\title{
STRING VERSUS EINSTEIN FRAME IN AdS/CFT INDUCED QUANTUM DILATONIC BRANE-WORLD UNIVERSE
}

\author{
Shin'ichi Nojiri* \\ Department of Applied Physics, National Defence Academy, Hashirimizu Yokosuka 239-8686, \\ JAPAN
}

\author{
Octavio Obregon ${ }^{\dagger}$, Sergei D. Odintsov ${ }^{\ddagger}$ and Vladimir I. Tkach ${ }^{\S}$ \\ Instituto de Fisica de la Universidad de Guanajuato, Lomas del Bosque 103, Apdo. Postal E-143, \\ 37150 Leon, Gto., MEXICO
}

\begin{abstract}
AdS/CFT induced quantum dilatonic brane-world where 4d boundary is flat or de Sitter (inflationary) or Anti-de Sitter brane is considered. The classical brane tension is fixed but boundary QFT produces the effective brane tension via the account of corresponding conformal anomaly induced effective action. This results in inducing of brane-worlds in accordance with AdS/CFT set-up as warped compactification. The explicit, independent construction of quantum induced dilatonic brane-worlds in two frames: string and Einstein one is done. It is demonstrated their complete equivalency for all quantum cosmological brane-worlds under discussion, including several examples of classical brane-world black holes. This is different from quantum corrected 4d dilatonic gravity where de Sitter solution exists in Einstein but not in Jordan (string) frame. The role of quantum corrections on massive graviton perturbations around Anti-de Sitter brane is briefly discussed.
\end{abstract}

98.80.Hw,04.50.+h,11.10.Kk,11.10.Wx

Typeset using REVTEX

\footnotetext{
*Electronic address: nojiri@cc.nda.ac.jp

†Electronic Address: octavio@ifug3.ugto.mx

${ }^{\ddagger}$ On leave from Tomsk State Pedagogical University, 634041 Tomsk, RUSSIA. Electronic address: odintsov@ifug5.ugto.mx

§Electronic Address: vladimir@ifug3.ugto.mx
} 


\section{INTRODUCTION}

Brane-worlds are alternative to the standard Kaluza-Klein compactification. They naturally lead to the following nice features of mutli-dimensional theory like trapping of $4 \mathrm{~d}$ gravity on the brane [1], resolution of hierarchy problem and possibly resolution of cosmological constant problem. Different aspects of brane-world cosmology (for very incomplete list of references see [2,3]) are under very active investigation.

The essential element of original brane-world models is the presence in the theory of two free parameters (bulk cosmological constant and brane tension, or brane cosmological constant). These parameters are fine-tuned (up to some extent) in order to construct the successful classical brane-world. This is most standard prescription which may be not completely satisfactory if one wishes to have the dynamical mechanism of brane tension origin.

From another side, one can fix the classical action on AdS-like space from the very beginning with the help of surface terms added in accordance with AdS/CFT correspondence [4. Such terms should make the variational procedure to be well-defined and also they should eliminate the leading divergence of the action. Brane tension is not considered as free parameter anymore but it is fixed by the condition of finiteness of spacetime when brane goes to infinity. In this case, as parameters are fixed the consistent brane-world scenario is impossible, as a rule. However, other parameters may improve the situation when quantum effects are taken into account. Taking quantum CFT (including quantum gravity!) on the brane one adds its contribution (the corresponding conformal anomaly induced effective action) to the total action. As a result, it changes the brane tension, the quantum induced brane-world occurs as it has been discovered in refs. [5,60. Actually, this represents the embedding of warped compactification (brane-worlds) to AdS/CFT correspondence, hence one gets AdS/CFT induced quantum brane-worlds [5,6] where 4d boundary may be flat or de Sitter or Anti-de Sitter spacetime. This is clearly the dynamical mechanism to get curved brane-world. It is easily generalized for the presence of non-trivial dilaton, i.e. AdS/CFT induced quantum dilatonic brane-worlds occur [7]. In other words, brane-worlds are the consequence of the presence of quantum fields on the brane in accord with AdS/CFT set-up. Moreover, such induced dilatonic brane-worlds are even more related with AdS/CFT correspondence as $5 \mathrm{~d}$ dilatonic gravity represents the bosonic sector of $5 \mathrm{~d}$ gauged supergravity (special parametrization). Even more, the dynamical determination of $4 \mathrm{~d}$ dilaton occurs.

In the study of quantum induced brane-worlds, in the same way as for any other dilatonic gravity the following question appears: which frame to work with is the physical one? There are two convenient frames: string (or Jordan) one where scalar curvature explicitly couples with dilaton and Einstein frame where scalar curvature does not couple with dilaton. Basically speaking, one should expect that results obtained in these two frames are not equivalent.

Indeed, in QFT the choice of different variables and (or) form of action corresponds to different parametrizations. QFT results are parametrization dependent, only S-matrix is gauge and parametrization independent. (Even the quantization procedure (for review, see [8]) is parametrization dependent.) As usually the consideration is one-loop ,one should expect in many cases the explicit parametrization dependence. Moreover, it is known that even for classical dilatonic gravity the (singular) solution may exist in only one parametriza- 
tion. Hence, the question of frame dependence should be carefully analyzed for all solutions at hands. This is the main purpose of the present work: to compare string frame quantum induced dilatonic brane-worlds with their analogs in Einstein frame.

In the next section as the simple example, $4 \mathrm{~d}$ dilatonic (Brans-Dicke) theory with large $N$ quantum spinor corrections is considered. In the Einstein frame where spinor is dilaton coupled one the de Sitter Universe solution with decaying dilaton exists. Working with the same theory in string (Jordan) frame where spinor is getting minimal, one finds that above solution does not exist. Hence, it is shown that two frames in 4d dilatonic gravity with quantum corrections are not equivalent.

In third section we consider $5 \mathrm{~d}$ dilatonic gravity action with $4 \mathrm{~d}$ boundary term induced by conformal anomaly of brane, dilaton coupled spinor. Explicit examples of de Sitter, flat and Anti-de Sitter dilatonic branes are constructed in Einstein frame. The dynamical mechanism to determine the dilaton on the brane is presented. In section four the same investigation is done in string frame. Brane spinor is now minimal. The same AdS/CFT induced quantum brane-worlds are proven to exist. Hence, for quantum corrected cosmological dilatonic braneworlds one has the equivalency of string and Einstein frames.

In fifth section the equivalency of string and Einstein frames is demonstrated for number of classical dilatonic brane-world black holes. In section six some remarks on massive graviton modes around dilatonic AdS4 brane are made. The role of brane quantum corrections for massive graviton modes is clarified. Brief summary and some outlook are given in final section.

\section{JORDAN AND EINSTEIN FRAMES FOR 4D QUANTUM CORRECTED DILATONIC GRAVITY}

In the study of dilatonic gravities the interesting question appears: which frame among few possible ones is the physical one? Basically speaking, there are two convenient frames to work with: string (or Jordan) frame and Einstein frame. These two are related by conformal transformation. The best known example is provided by the standard Brans-Dicke theory (with matter). The 4-dimensional action in the Jordan frame is:

$$
S_{B D}=\frac{1}{16 \pi} \int d^{4} x \sqrt{-g}\left[\phi R-\frac{\omega}{\phi}\left(\nabla_{\mu} \phi\right)\left(\nabla^{\mu} \phi\right)\right]+S_{M},
$$

where $\phi$ is the Brans-Dicke (dilaton) field with $\omega$ being the coupling constant and $S_{M}$ is the matter action.

Performing the following conformal transformation and a redefinition of the scalar field

$$
\tilde{g}_{\mu \nu}=G \phi g_{\mu \nu}, \quad \tilde{\phi}=\sqrt{\frac{2 \omega+3}{16 \pi G}} \ln (G \phi), \quad 2 \omega+3>0 .
$$

one gets the action in the Einstein frame

$$
S=\int d^{4} x \sqrt{-\tilde{g}(x)}\left[\frac{\tilde{R}}{16 \pi G}-\frac{1}{2}\left(\tilde{\nabla}_{\mu} \tilde{\phi}\right)\left(\tilde{\nabla}^{\mu} \tilde{\phi}\right)+\exp (A \tilde{\phi}) L_{M}(\tilde{g})\right],
$$


where $A=-8 \sqrt{\frac{\pi G}{2 \omega+3}}$. It is expected that these two actions (at least for regular solutions) should lead to equivalent results. However, the explicit consideration shows that it is not always so (for a review, see [9]). That is why it was argued in ref. [9] that it is Einstein frame which is physical one. Of course, such state of affairs is not satisfactory.

In quantum field theory the choice of different variables corresponds to different parametrizations. It is known that generally speaking it leads to parametrization dependent results: it is only S-matrix should be the same in different parametrizations. Of course, this should be true only in complete theory where account of all loops is taken. As usually the consideration is one-loop, one should expect parametrization dependence already at one-loop.

Let us consider the explicit example in Einstein frame where quantum corrections are taken into account. As matter Lagrangian we take the one associated with $N$ massless (Dirac) spinors, i.e. $L_{M}=\sum_{i=1}^{N} \bar{\psi}_{i} \gamma^{\mu} \nabla_{\mu} \psi^{i}$. There is no problem to add other types of matter (say scalar or vector fields). The above choice is made only for the sake of simplicity.

We shall make use of the EA formalism (for an introduction, see [10]). The corresponding 4d anomaly-induced EA for dilaton coupled scalars, vectors and spinors has been found in Refs. [11].

Hence, starting from the theory with the action (no classical background spinors)

$$
S=\int d^{4} x \sqrt{-g}\left[\frac{R}{16 \pi G}-\frac{1}{2}\left(\nabla_{\mu} \phi\right)\left(\nabla^{\mu} \phi\right)+\exp (A \phi) \sum_{i=1}^{N} \bar{\psi}_{i} \gamma^{\mu} \nabla_{\mu} \psi^{i}\right],
$$

we will discuss FRW type cosmologies

$$
d s^{2}=-d t^{2}+a(t)^{2} d l^{2},
$$

where $d l^{2}$ is the line metric element of a 3-dimensional flat space.

The computation of the anomaly-induced EA for the dilaton coupled spinor field has been done in [11, and the result, in the non-covariant local form, reads:

$$
\begin{aligned}
& W=\int d^{4} x \sqrt{-\bar{g}}\left\{b \bar{F} \sigma_{1}+2 b^{\prime} \sigma_{1}\left[\square^{2}+2 \bar{R}^{\mu \nu} \bar{\nabla}_{\mu} \bar{\nabla}_{\nu}-\frac{2}{3} \bar{R} \square+\frac{1}{3}\left(\bar{\nabla}^{\mu} \bar{R}\right) \bar{\nabla}_{\mu}\right] \sigma_{1}\right. \\
& \left.+b^{\prime} \sigma_{1}\left(\bar{G}-\frac{2}{3} \square \bar{R}\right)-\frac{1}{18}\left(b+b^{\prime}\right)\left[\bar{R}-6 \square \sigma_{1}-6\left(\bar{\nabla}_{\mu} \sigma_{1}\right)\left(\bar{\nabla}^{\mu} \sigma_{1}\right)\right]^{2}\right\},
\end{aligned}
$$

where $\sigma_{1}=\sigma+A \phi / 3$, the square of the Weyl tensor is given by $F=R_{\mu \nu \rho \sigma} R^{\mu \nu \rho \sigma}-2 R_{\mu \nu} R^{\mu \nu}+$ $\frac{1}{3} R^{2}$ and Gauss-Bonnet invariant is $G=R_{\mu \nu \rho \sigma} R^{\mu \nu \rho \sigma}-4 R_{\mu \nu} R^{\mu \nu}+R^{2}$. For Dirac spinors $b=\frac{3 N}{60(4 \pi)^{2}}, b^{\prime}=-\frac{11 N}{360(4 \pi)^{2}}$.

Then we find the following Einstein frame, quantum-corrected solution whose metric is expressed in Jordan frame as

$$
\begin{aligned}
d s_{J}^{2} & =a_{J}^{2}(\eta)\left(-d \eta^{2}+d l^{2}\right) \\
a_{J}^{2}(\eta) & =\mathrm{e}^{-\frac{\phi}{\sqrt{\frac{2 \omega+3}{16 G}}}} a^{2}(\eta) \\
& =a_{0} \eta^{-2 \zeta} \\
\zeta & \equiv \frac{1}{2 H_{1}} \sqrt{\frac{16 \pi G}{2 \omega+3}}+1
\end{aligned}
$$




$$
\begin{aligned}
& =\frac{1}{\sqrt{2 \omega+3}\left\{-\frac{3}{16} \sqrt{2 \omega+3} \pm \sqrt{\frac{9}{256}(2 \omega+3)-\frac{1}{6}}\right\}}+1 \\
& =-\frac{1}{8} \mp \sqrt{\frac{81}{64}-\frac{6}{2 \omega+3}} .
\end{aligned}
$$

Here $a_{0}$ is an arbitrary constant. On the other hand, one finds the dilaton field $\phi_{J}$ in the Jordan frame as

$$
\phi=\phi_{0} \eta^{\frac{1}{H_{1}} \sqrt{\frac{16 \pi G}{2 \omega+3}}}=\phi_{0} \eta^{2(\zeta-1)}, \quad \phi_{0}=\frac{1}{a_{0} G} .
$$

Let us analyze the equations of motion in the Jordan frame (for the form of transformation to string frame see section 5). The variations over $\phi$ and $\sigma$ give the following equations:

$$
\begin{aligned}
0= & 6\left(\sigma^{\prime \prime}+{\sigma^{\prime}}^{2}\right) \mathrm{e}^{2 \sigma}-\frac{\omega \phi^{\prime 2}}{\phi^{2}} \mathrm{e}^{2 \sigma}-2 \omega\left(\frac{\phi^{\prime} \mathrm{e}^{2 \sigma}}{\phi}\right), \\
0= & \frac{2}{16 \pi}\left(6\left(\sigma^{\prime \prime}+{\sigma^{\prime}}^{2}\right)+\frac{\omega \phi^{\prime 2}}{\phi}\right) \mathrm{e}^{2 \sigma}+\frac{6\left(\mathrm{e}^{2 \sigma}\right)^{\prime \prime}-12\left(\sigma^{\prime} \mathrm{e}^{2 \sigma}\right)^{\prime}}{16 \pi} \\
& +4 b^{\prime} \sigma^{\prime \prime \prime \prime}-4\left(b+b^{\prime}\right)\left\{\left(\sigma^{\prime \prime}-{\sigma^{\prime}}^{2}\right)^{\prime \prime}+2\left(\sigma^{\prime}\left(\sigma^{\prime \prime}-{\sigma^{\prime}}^{2}\right)\right)^{\prime}\right\} .
\end{aligned}
$$

Here $^{\prime} \equiv \frac{d}{d \eta}$. We can check that the solution (7) and (8) does not satisfy (9). If the solution in the Jordan frame would be equivalent to that in the Einstein frame even in the quantum level, we should have $\sigma_{1}=\sigma_{J} \equiv \ln a_{J}$ but we have $\sigma_{1}=\sigma+\frac{A \phi}{3}=\sigma-\frac{4}{3} \ln G \phi_{J}$ and $\sigma_{J}=\sigma-\frac{1}{2} \ln G \phi_{J}$. This is an origin of the inequivalence. Thus, it is demonstrated that for the Universe model under consideration the Jordan and Einstein frames in $4 \mathrm{~d}$ dilatonic gravity with quantum corrections are not equivalent. Different parametrizations lead to different results (parametrization choice dependence). The physical results are expecting to be the same only for S-matrix in full theory (non-perturbative regime).

\section{INFLATIONARY DILATONIC BRANE-WORLD UNIVERSE IN EINSTEIN FRAME}

In this section we present the review of quantum induced dilatonic brane-worlds found in ref. [7]. The model is discussed in Einstein frame and using euclidean notations. This scenario represents the extension to non-constant dilaton case the earlier scenario of refs. [5.6] where quantum brane-worlds were realized in frames of AdS/CFT correspondence, by adding quantum CFT on the brane to effective action.

We start with Euclidean signature action $S$ which is the sum of the Einstein-Hilbert action $S_{\mathrm{EH}}$ including dilaton $\phi$ with potential $V(\phi)=\frac{12}{l^{2}}+\Phi(\phi)$, the Gibbons-Hawking surface term $S_{\mathrm{GH}}$, the surface counter term $S_{1}$ 円

\footnotetext{
${ }^{1}$ We use the following curvature conventions:
} 


$$
\begin{aligned}
S & =S_{\mathrm{EH}}+S_{\mathrm{GH}}+2 S_{1}, \\
S_{\mathrm{EH}} & =\frac{1}{16 \pi G} \int d^{5} x \sqrt{g_{(5)}}\left(R_{(5)}-\frac{1}{2} \nabla_{\mu} \phi \nabla^{\mu} \phi+\frac{12}{l^{2}}+\Phi(\phi)\right), \\
S_{\mathrm{GH}} & =\frac{1}{8 \pi G} \int d^{4} x \sqrt{g_{(4)}} \nabla_{\mu} n^{\mu}, \\
S_{1} & =-\frac{1}{16 \pi G} \int d^{4} \sqrt{g_{(4)}}\left(\frac{6}{l}+\frac{l}{4} \Phi(\phi)\right) .
\end{aligned}
$$

Here the quantities in the 5 dimensional bulk spacetime are specified by the suffices (5) and those in the boundary 4 dimensional spacetime are specified by (4). The factor 2 in front of $S_{1}$ in (11) is coming from that we have two bulk regions which are connected with each other by the brane. It is clear that above representation corresponds to Einstein frame. In (13), $n^{\mu}$ is the unit vector normal to the boundary.

\section{A. Bulk solutions}

In this subsection, we find some explicit solutions in the bulk space.

We now assume the metric in the following form

$$
d s^{2}=f(y) d y^{2}+y \sum_{i, j=0}^{3} \hat{g}_{i j}\left(x^{k}\right) d x^{i} d x^{j},
$$

and $\phi$ depends only on $y: \phi=\phi(y)$. Here $\hat{g}_{i j}$ is the metric of the Einstein manifold, which is defined by $r_{i j}=k \hat{g}_{i j}$, where $r_{i j}$ is the Ricci tensor constructed with $\hat{g}_{i j}$ and $k$ is a constant. Then we obtain the following equations of motion in the bulk:

$$
\begin{aligned}
& 0=\frac{3}{2 y^{2}}-\frac{2 k f}{y}-\frac{1}{4}\left(\frac{d \phi}{d y}\right)^{2}-\left(\frac{6}{l^{2}}+\frac{1}{2} \Phi(\phi)\right) f \\
& 0=\frac{d}{d y}\left(\frac{y^{2}}{\sqrt{f}} \frac{d \phi}{d y}\right)+\Phi^{\prime}(\phi) y^{2} \sqrt{f}
\end{aligned}
$$

It is convenient to introduce the new coordinate $z$

$$
z=\int d y \sqrt{f(y)} .
$$

$$
\begin{aligned}
R & =g^{\mu \nu} R_{\mu \nu} \\
R_{\mu \nu} & =R_{\mu \lambda \nu}^{\lambda} \\
R_{\mu \rho \nu}^{\lambda} & =-\Gamma_{\mu \rho, \nu}^{\lambda}+\Gamma_{\mu \nu, \rho}^{\lambda}-\Gamma_{\mu \rho}^{\eta} \Gamma_{\nu \eta}^{\lambda}+\Gamma_{\mu \nu}^{\eta} \Gamma_{\rho \eta}^{\lambda} \\
\Gamma_{\mu \lambda}^{\eta} & =\frac{1}{2} g^{\eta \nu}\left(g_{\mu \nu, \lambda}+g_{\lambda \nu, \mu}-g_{\mu \lambda, \nu}\right) .
\end{aligned}
$$


By solving $y$ with respect to $z$, we obtain the warp factor $l^{2} \mathrm{e}^{2 \hat{A}(z, k)}=y(z)$. Here one assumes the metric of 5 dimensional space time as follows:

$$
d s^{2}=d z^{2}+\mathrm{e}^{2 A(z, \sigma)} \tilde{g}_{\mu \nu} d x^{\mu} d x^{\nu}, \quad \tilde{g}_{\mu \nu} d x^{\mu} d x^{\nu} \equiv l^{2}\left(d \sigma^{2}+d \Omega_{3}^{2}\right) .
$$

where $d \Omega_{3}^{2}$ corresponds to the metric of 3 dimensional unit sphere. Suppose that $A(z, \sigma)$ can be decomposed into the sum of $z$-dependent part $\hat{A}(z)$ and $\sigma$-dependent part and therefore $l^{2} \mathrm{e}^{2 \hat{A}(z)} \hat{g}_{\mu \nu}=\mathrm{e}^{2 A(z, \sigma)} \tilde{g}_{\mu \nu}$. Then for the unit sphere $(k=3)$

$$
A(z, \sigma)=\hat{A}(z, k=3)-\ln \cosh \sigma
$$

for the flat Euclidean space $(k=0)$

$$
A(z, \sigma)=\hat{A}(z, k=0)+\sigma,
$$

and for the unit hyperboloid $(k=-3)$

$$
A(z, \sigma)=\hat{A}(z, k=-3)-\ln \sinh \sigma
$$

When $\Phi(\phi)=0$, there exists the following AdS-like solution of the equations of motion 14

$$
\begin{aligned}
d s^{2} & =f(y) d y^{2}+y \sum_{i, j=0}^{d-1} \hat{g}_{i j}\left(x^{k}\right) d x^{i} d x^{j} \\
f & =\frac{d(d-1)}{4 y^{2} \lambda^{2}\left(1+\frac{c^{2}}{2 \lambda^{2} y^{d}}+\frac{k d}{\lambda^{2} y}\right)} \\
\phi & =c \int d y \sqrt{\frac{d(d-1)}{4 y^{d+2} \lambda^{2}\left(1+\frac{c^{2}}{2 \lambda^{2} y^{d}}+\frac{k d}{\lambda^{2} y}\right)}} .
\end{aligned}
$$

Here $\lambda^{2}=\frac{12}{l^{2}}$.

When $\Phi(\phi) \neq 0$, by using (16) and (17), one can delete $f$ from the equations and we obtain an equation that contains only the dilaton field $\phi$ :

$$
\begin{aligned}
0= & \left\{\frac{5 k}{2}-\frac{k}{4} y^{2}\left(\frac{d \phi}{d y}\right)^{2}+\left(\frac{3}{2} y-\frac{y^{3}}{6}\left(\frac{d \phi}{d y}\right)^{2}\right)\left(\frac{6}{l^{2}}+\frac{1}{2} \Phi(\phi)\right)\right\} \frac{d \phi}{d y} \\
& +\frac{y^{2}}{2}\left(\frac{2 k}{y}+\frac{6}{l^{2}}+\frac{1}{2} \Phi(\phi)\right) \frac{d^{2} \phi}{d y^{2}}+\left(\frac{3}{4}-\frac{y^{2}}{8}\left(\frac{d \phi}{d y}\right)^{2}\right) \Phi^{\prime}(\phi) .
\end{aligned}
$$

We now consider a solvable case where

$$
\frac{6}{l^{2}}+\frac{1}{2} \Phi(\phi)=-\frac{2 k}{y} .
$$

The explicit form, or $\phi$ dependence, of $\Phi(\phi)$ can be determined after solving the equations of motion as the following 


$$
\phi= \pm \sqrt{6} \ln \left(m^{2} y\right)
$$

Here $m^{2}$ is a constant of the integration. The explicit form of $\Phi(\phi)$ is:

$$
\Phi(\phi)=-\frac{12}{l^{2}}-4 k m^{2} \mathrm{e}^{\mp \frac{\phi}{\sqrt{6}}} .
$$

One can also find that Eq.(16) is trivially satisfied. Integrating (17), we obtain

$$
f=\frac{1}{-\frac{2 k y}{9}+\frac{f_{0}}{y^{2}}} .
$$

Here $f_{0}$ is a constant of the integration and $f_{0}$ should be positive in order that $f$ is positive for large $y$. There is a (curvature) singularity at $y=0$. One should also note that when $k>0$, the horizon appears at

$$
y^{3}=y_{0}^{3} \equiv \frac{9 f_{0}}{2 k}
$$

and we find

$$
y \leq y_{0}
$$

\section{B. Brane solutions}

In this subsection, we investigate if there is a solution with brane including the quantum correction from $N$ massless brane Majorana spinors coupled with the dilaton. For simplicity, only the case that the potential is constant.

On the brane, one obtains the following equations by the variations over $A$ and $\phi$ :

$$
\begin{aligned}
& 0=\frac{48 l^{4}}{16 \pi G}\left(\partial_{z} A-\frac{1}{l}-\frac{l}{24} \Phi(\phi)\right) \mathrm{e}^{4 A}, \\
& 0=-\frac{l^{4}}{8 \pi G} \mathrm{e}^{4 A} \partial_{z} \phi-\frac{l^{5}}{32 \pi G} \mathrm{e}^{4 A} \Phi^{\prime}(\phi) .
\end{aligned}
$$

With (27) and the solution (28), these equations look

$$
\begin{aligned}
& 0=\frac{1}{2 R^{2}} \sqrt{\frac{f_{0}}{R^{4}}-\frac{2 k R^{2}}{9}}-\frac{1}{2 l}+\frac{k l}{3 R^{2}}, \\
& 0=\sqrt{\frac{f_{0}}{R^{4}}-\frac{2 k R^{2}}{9}+\frac{k l}{k l}} .
\end{aligned}
$$

Here we assume that the brane lies at $y=y_{0}$ or $z=z_{0}$. The radius $R$ of the brane is defined by $R=\mathrm{e}^{\hat{A}\left(z_{0}\right)}$. Eq.(34) tells that $k \leq 0$ but by combining (33) and (34), we find $R^{2}=\frac{k l^{2}}{2}$. Then there is no consistent classical solution.

We now consider the case that the matter on the brane is some QFT like QED or QCD. Of course, such a theory is classically conformally invariant one. As an explicit example in 
order to be able to apply large $N$-expansion we suppose that dominant contribution is due to $N$ massless Majorana spinors coupled with the dilaton, whose action is given by

$$
S=\int \sqrt{g_{(4)}} \mathrm{e}^{a \phi} \sum_{i=1}^{N} \bar{\Psi}_{i} \gamma^{\mu} D_{\mu} \Psi_{i} .
$$

The case of minimal spinor coupling corresponds to the choice $a=0$. Note that from Brans-Dicke theory consideration one knows that for Einstein frame the non-minimal dilaton coupling with the matter is the typical case. Then the trace anomaly induced action $W$ has the following form [1]:

$$
\begin{aligned}
W= & b \int d^{4} x \sqrt{\widetilde{g}} \widetilde{F} A_{1} \\
& +b^{\prime} \int d^{4} x\left\{A_{1}\left[2 \square^{2}+\widetilde{R}_{\mu \nu} \widetilde{\nabla}_{\mu} \widetilde{\nabla}_{\nu}-\frac{4}{3} \widetilde{R} \square^{2}+\frac{2}{3}\left(\widetilde{\nabla}^{\mu} \widetilde{R}\right) \widetilde{\nabla}_{\mu}\right] A_{1}\right. \\
& \left.+\left(\widetilde{G}-\frac{2}{3} \square \widetilde{R}\right) A_{1}\right\} \\
& -\frac{1}{12}\left\{b^{\prime \prime}+\frac{2}{3}\left(b+b^{\prime}\right)\right\} \int d^{4} x\left[\widetilde{R}-6 \square A_{1}-6\left(\widetilde{\nabla}_{\mu} A_{1}\right)\left(\widetilde{\nabla}^{\mu} A_{1}\right)\right]^{2} .
\end{aligned}
$$

Here

$$
A_{1}=A+\frac{a \phi}{3}
$$

and

$$
b=\frac{3 N}{60(4 \pi)^{2}}, \quad b^{\prime}=-\frac{11 N}{360(4 \pi)^{2}} .
$$

We also choose $b^{\prime \prime}=0$ as it may be changed by finite renormalization of classical gravitational action. In (36), one chooses the 4 dimensional boundary metric as

$$
g_{(4)} \mu \nu=\mathrm{e}^{2 A} \tilde{g}_{\mu \nu}
$$

and we specify the quantities given by $\tilde{g}_{\mu \nu}$ by using $\sim G(\tilde{G})$ and $F(\tilde{F})$ are the Gauss-Bonnet invariant and the square of the Weyl tensor, which are given as

$$
\begin{aligned}
& G=R^{2}-4 R_{i j} R^{i j}+R_{i j k l} R^{i j k l}, \\
& F=\frac{1}{3} R^{2}-2 R_{i j} R^{i j}+R_{i j k l} R^{i j k l},
\end{aligned}
$$

For simplicity, we consider a constant potential $(\Phi(\phi)=0)$ case. Then brane equations are

$$
\begin{aligned}
0= & \frac{48 l^{4}}{16 \pi G}\left(\partial_{z} A-\frac{1}{l}\right) \mathrm{e}^{4 A}+b^{\prime}\left(4 \partial_{\sigma}^{4} A_{1}-16 \partial_{\sigma}^{2} A_{1}\right) \\
& -4\left(b+b^{\prime}\right)\left(\partial_{\sigma}^{4} A_{1}+2 \partial_{\sigma}^{2} A_{1}-6\left(\partial_{\sigma} A_{1}\right)^{2} \partial_{\sigma}^{2} A_{1}\right) \\
0= & -\frac{l^{4}}{8 \pi G} \mathrm{e}^{4 A} \partial_{z} \phi+\frac{4}{3} a b^{\prime}\left(4 \partial_{\sigma}^{4} A_{1}-16 \partial_{\sigma}^{2} A_{1}\right) .
\end{aligned}
$$


Then one gets

$$
\begin{aligned}
& 0=\frac{1}{\pi G l}\left\{\sqrt{1+\frac{k l^{2}}{3 R^{2}}+\frac{l^{2} c^{2}}{24 R^{8}}}-1\right\} R^{4}+8 b^{\prime}, \\
& 0=-\frac{c}{8 \pi G}+32 a b^{\prime} .
\end{aligned}
$$

Note that for minimal spinor coupling the second equation does not have a solution. Eq.(44) can be solved with respect to $c$ :

$$
c=32 \times 8 \pi G a b^{\prime},
$$

but the boundary value $\phi_{0}$ of $\phi$ becomes a free parameter.

We should also note that in the classical case that $b^{\prime}=0$, there is no solution for (43) and (44). From Eq.(44), we find $c=0$ if $b^{\prime}=0$. Then if we put $c=0$ and $b^{\prime}=0$ in (43), there is no solution.

When the dilaton vanishes $(c=0)$ and the brane is the unit sphere $(k=3)$, the equation (43) reproduces the result of ref. [6] for $\mathcal{N}=4 S U(N)$ super Yang-Mills theory in case of the large $N$ limit where $b^{\prime}$ is replaced by $-\frac{N^{2}}{4(4 \pi)^{2}}$ :

$$
\frac{R^{3}}{l^{3}} \sqrt{1+\frac{R^{2}}{l^{2}}}=\frac{R^{4}}{l^{4}}+\frac{G N^{2}}{8 \pi l^{3}} .
$$

Let us define a function $F(R, c)$ as

$$
F(R, c) \equiv \frac{1}{\pi G l}\left(\sqrt{1+\frac{k l^{2}}{3 R^{2}}+\frac{l^{2} c^{2}}{24 R^{8}}}-1\right) R^{4}
$$

which appears in the r.h.s. in (43).

For the $k>0$ case, $F(R, c)$ has a minimum at $R=R_{0}$, where $R_{0}$ is defined by

$$
0=\frac{8 k l^{2}}{3 R_{0}^{2}}+\frac{k^{2} l^{4}}{R_{0}^{4}}-\frac{2 l^{2} c^{2}}{3 R_{0}^{8}} .
$$

When $k>0$, there is only one solution for $R_{0}$. Therefore $F(R, c)$ in the case of $k>0$ (sphere case) is a monotonically increasing function of $R$ when $R>R_{0}$ and a decreasing function when $R<R_{0}$. Since $F(R, c)$ is clearly a monotonically increasing function of $c$, we find for $k>0$ and $b^{\prime}<0$ case that $R$ decreases when $c$ increases if $R>R_{0}$, that is, the non-trivial dilaton makes the radius smaller. We can also find that there is no solution for $R$ in (43) for very large $|c|$.

We can consider the $k<0$ case. When $c=0$, there is no solution for $R$ in (43). We can find, however, there is a solution if $|c|$ is large enough:

$$
\frac{|c|}{\pi G \sqrt{24}}>-8 b^{\prime} \text {. }
$$

Hence, for constant bulk potential there is the possibility of quantum creation of a $4 \mathrm{~d}$ de Sitter or a $4 \mathrm{~d}$ hyperbolic brane living in $5 \mathrm{~d}$ AdS bulk space. This occurs even for not exactly conformal invariant quantum brane matter. This finishes our consideration of quantum induced dilatonic brane-worlds in Einstein frame. 


\section{QUANTUM INDUCED DILATONIC BRANE-WORLDS IN STRING FRAME.}

We now transform the brane-world action in the Einstein frame (see (11)) into the Jordan frame. If we consider the scale transformation

$$
g_{\mu \nu} \rightarrow \mathrm{e}^{\rho} g_{\mu \nu}
$$

with the choice

$$
\mathrm{e}^{\left(\frac{D}{2}-1\right) \rho}=\alpha \phi, \quad(\alpha \text { is a constant })
$$

we find that the actions (12), (13) and (14) are transformed as

$$
\begin{aligned}
S_{\mathrm{EH}}= & \frac{1}{16 \pi G} \int d^{5} x \sqrt{g_{(5)}}\left(\alpha \phi R_{(5)}+\frac{4 \alpha}{3 \phi} \nabla_{\mu} \phi \nabla^{\mu} \phi-\frac{\alpha}{2} \phi \nabla_{\mu} \phi \nabla^{\mu} \phi\right. \\
& \left.+\left(\frac{12}{l^{2}}+\Phi(\phi)\right)(\alpha \phi)^{\frac{5}{3}}\right), \\
S_{\mathrm{GH}}= & \frac{1}{8 \pi G} \int d^{4} x \alpha \phi \sqrt{g_{(4)}} \nabla_{\mu} n^{\mu}, \\
S_{1}= & -\frac{1}{16 \pi G} \int d^{4} x(\alpha \phi)^{\frac{4}{3}} \sqrt{g_{(4)}}\left(\frac{6}{l}+\frac{l}{4} \Phi(\phi)\right) .
\end{aligned}
$$

\section{A. Bulk solution in the string frame}

In the bulk, the variation over $\phi$ gives the following equation of motion:

$$
\begin{aligned}
0= & \alpha R_{(5)}-\frac{4 \alpha}{3 \phi^{2}} \partial_{\mu} \phi \partial^{\mu} \phi-\frac{\alpha}{2} \partial_{\mu} \phi \partial^{\mu} \phi+\frac{5}{3}\left(\frac{12}{l^{2}}+\Phi(\phi)\right) \alpha^{\frac{5}{3}} \phi^{\frac{2}{3}} \\
& +\Phi^{\prime}(\phi)(\alpha \phi)^{\frac{5}{3}}-\frac{8 \alpha}{3} \nabla_{\mu}\left(\frac{1}{\phi} \partial^{\mu} \phi\right)+\alpha \nabla_{\mu}\left(\phi \partial^{\mu} \phi\right) .
\end{aligned}
$$

On the other hand, the variation over the metric $g^{\mu \nu}$ gives

$$
\begin{aligned}
0= & -\frac{1}{2}\left(\alpha \phi R_{(5)}+\frac{4 \alpha}{3 \phi} \partial_{\mu} \phi \partial^{\mu} \phi-\frac{\alpha}{2} \phi \partial_{\mu} \phi \partial^{\mu} \phi+\left(\frac{12}{l^{2}}+\Phi(\phi)\right)(\alpha \phi)^{\frac{5}{3}}\right) g_{(5) \mu \nu} \\
& +\alpha \phi R_{(5) \mu \nu}-\alpha \nabla_{\mu} \partial_{\nu} \phi+\alpha g_{(5) \mu \nu} \square \phi \\
& +\frac{4 \alpha}{3 \phi} \partial_{\mu} \phi \partial_{\nu} \phi-\frac{\alpha}{2} \partial_{\mu} \phi \partial_{\nu} \phi .
\end{aligned}
$$

Thus, one gets the bulk equations of motion in string frame. Using (56), we have

$$
\begin{aligned}
0= & -\frac{3}{2}\left(\alpha \phi R_{(5)}+\frac{4 \alpha}{3 \phi} \partial_{\mu} \phi \partial^{\mu} \phi-\frac{\alpha}{2} \phi \partial_{\mu} \phi \partial^{\mu} \phi\right) \\
& -\frac{5}{2}\left(\frac{12}{l^{2}}+\Phi(\phi)\right)(\alpha \phi)^{\frac{5}{3}}+4 \alpha \square \phi .
\end{aligned}
$$


Substituting (57) into (55) and (56), one obtains

$$
\begin{aligned}
0= & \alpha \nabla_{\mu}\left(\phi \partial^{\mu} \phi\right)+\Phi^{\prime}(\phi)(\alpha \phi)^{\frac{5}{3}} \\
0= & -\alpha \nabla_{\mu} \partial_{\nu} \phi-\frac{\alpha}{3} g_{(5) \mu \nu} \square \phi+\alpha \phi R_{(5) \mu \nu} \\
& +\frac{1}{3}\left(\frac{12}{l^{2}}+\Phi(\phi)\right)(\alpha \phi)^{\frac{5}{3}} g_{(5) \mu \nu}+\frac{4 \alpha}{3 \phi} \partial_{\mu} \phi \partial_{\nu} \phi-\frac{\alpha}{2} \phi \partial_{\mu} \phi \partial_{\nu} \phi .
\end{aligned}
$$

First, let us consider $\Phi(\phi)=0$ case. In the Einstein frame, the solution is given by (23). The metric $g_{(5) \mu \nu}^{\mathrm{J}}$ in the Jordan frame is obtained with the help of (50) and (51), or more explicitly

$$
\begin{aligned}
g_{(5) \mu \nu}^{\mathrm{J}} d x^{\mu} d x^{\nu} & =(\alpha \phi)^{-\frac{2}{3}}\left(f(y) d y^{2}+y \sum_{i, j=0}^{3} \hat{g}_{i j}\left(x^{k}\right) d x^{i} d x^{j}\right) \\
f & =\frac{l^{2}}{4 y^{2}\left(1+\frac{c^{2} l^{2}}{24 y^{4}}+\frac{k l^{2}}{3 y}\right)} \\
\phi & =c \int d y \frac{l}{2 \sqrt{y^{6}\left(1+\frac{c^{2} l^{2}}{24 y^{4}}+\frac{k l^{2}}{3 y}\right)}} .
\end{aligned}
$$

One can check directly that the metric (60) satisfies Eqs.(58) and (59). Although the classical bulk solution in the Einstein frame is equivalent to the one in the Jordan frame, the physical interpretation of the spacetime is changed due to the factor of $(\alpha \phi)^{-\frac{2}{3}}$. Since the transformation is conformal, the causal structure of the spacetime is not changed, especially the situation that there is a curvature singularity at $y=0$ is not changed. When $y \rightarrow \infty$, however, the spacetime is not asymptotically AdS but the metric behaves as

$$
g_{(5) \mu \nu}^{\mathrm{J}} d x^{\mu} d x^{\nu} \sim\left(-\frac{\alpha c l}{4}\right)^{-\frac{2}{3}}\left(\frac{l^{2}}{4 y^{\frac{2}{3}}} d y^{2}+y^{\frac{7}{3}} \sum_{i, j=0}^{3} \hat{g}_{i j}\left(x^{k}\right) d x^{i} d x^{j}\right) .
$$

If one defines a coordinate $z$ by

$$
z \equiv\left(-\frac{\alpha c l}{4}\right)^{-\frac{1}{3}} \frac{3 l}{4} y^{\frac{2}{3}}
$$

the metric in (61) is rewritten by

$$
g_{(5) \mu \nu}^{\mathrm{J}} d x^{\mu} d x^{\nu} \sim d z^{2}+\left(-\frac{\alpha c l}{4}\right)^{\frac{1}{2}}\left(\frac{4 z}{3 l}\right)^{\frac{7}{2}} \sum_{i, j=0}^{3} \hat{g}_{i j}\left(x^{k}\right) d x^{i} d x^{j} .
$$

Then the warp factor behaves as the power of $z$, instead of the exponential function in Einstein frame.

One can also consider the case that the dilaton potential $\frac{12}{l^{2}}+\Phi(\phi)$ is given by (27). Using the relation (50) and (51) between the Einstein frame and the Jordan frame, from (26) and (28), we find the following solution: 


$$
\begin{aligned}
g_{(5) \mu \nu}^{\mathrm{J}} d x^{\mu} d x^{\nu} & =\left( \pm \alpha \sqrt{6} \ln \left(m^{2} y\right)\right)^{-\frac{2}{3}}\left(\frac{1}{-\frac{2 k y}{9}+\frac{f_{0}}{y^{2}}} d y^{2}+y \sum_{i, j=0}^{3} \hat{g}_{i j}\left(x^{k}\right) d x^{i} d x^{j}\right) \\
\phi & = \pm \sqrt{6} \ln \left(m^{2} y\right) .
\end{aligned}
$$

One can again check that the above solution satisfies Eqs.(58) and (59). Then the above result is equivalent with that in the Einstein frame. Comparing the obtained metric with that in the Einstein frame in (26) and (28), there appears the factor of the logarithmic function of $y$, coming from the conformal transformation. In other words, the interpretation of lenghts in both frames is different while solutions are equivalent.

\section{B. Brane solutions in the string frame}

Having proof of explicit equivalency of bulk solutions, one can analyze the brane. From the actions in (52), (53) and (54), the variation over $\phi$ gives the following equation on the boundary

$$
\begin{aligned}
0= & \frac{l^{4} \mathrm{e}^{4 A}}{8 \pi G}\left\{\left(\frac{8 \alpha}{3 \phi_{0}}-\alpha \phi_{0}\right) \partial_{z} \phi+8 \alpha \partial_{z} A\right. \\
& \left.-\frac{4 \alpha}{3}\left(\frac{6}{l}+\frac{l}{4} \Phi(\phi)\right)\left(\alpha \phi_{0}\right)^{\frac{1}{3}}-\frac{l}{4} \Phi^{\prime}(\phi)(\alpha \phi)^{\frac{4}{3}}\right\} .
\end{aligned}
$$

Here we choose the metric as in (19) and $\phi_{0}$ is the value of $\phi$ on the boundary. The variation over $A$ gives the following equation

$$
0=\frac{48 l^{4}}{16 \pi G} \mathrm{e}^{4 A}\left(\alpha \phi_{0} \partial_{z} A+\frac{\alpha}{3} \partial_{z} \phi-\frac{1}{6}\left(\frac{6}{l}+\frac{l}{4} \Phi(\phi)\right)\left(\alpha \phi_{0}\right)^{\frac{4}{3}}\right) .
$$

The coordinate $z$ and $A$ in the warp factor are related with those in the Einstein frame, $z_{E}$ and $A_{E}$ by

$$
d z_{E}=(\alpha \phi)^{\frac{1}{3}} d z, \quad A_{E}=A+\frac{1}{3} \ln (\alpha \phi) .
$$

Then Eqs. (65) and (66) are rewritten as

$$
\begin{aligned}
0= & \frac{l^{4} \mathrm{e}^{4 A_{E}}}{8 \pi G}\left\{-\partial_{z_{E}} \phi+\alpha\left(\alpha \phi_{0}\right)\left(8 \partial_{z_{E}} A_{E}-\frac{4 \alpha}{3}\left(\frac{6}{l}+\frac{l}{4} \Phi(\phi)\right)\right.\right. \\
& \left.\left.-\frac{l}{4} \Phi^{\prime}(\phi)\right)\right\} \\
0= & \frac{48 l^{4}}{16 \pi G} \mathrm{e}^{4 A_{E}}\left\{\partial_{z_{E}} A_{E}-\frac{1}{6}\left(\frac{6}{l}+\frac{l}{4} \Phi(\phi)\right)\right\} .
\end{aligned}
$$

Combining (68) and (69), we obtain

$$
0=\frac{l^{4} \mathrm{e}^{4 A_{E}}}{8 \pi G}\left\{-\partial_{z_{E}} \phi-\frac{l}{4} \Phi^{\prime}(\phi)\right\} .
$$


The obtained equations (69) and (70) are identical with the corresponding equations (31) and (32) without the quantum correction, respectively.

Choosing the metric of 5 dimensional space-time as in (19):

$$
d s^{2}=d z^{2}+\mathrm{e}^{2 A(z, \sigma)} \tilde{g}_{\mu \nu} d x^{\mu} d x^{\nu}, \quad \tilde{g}_{\mu \nu} d x^{\mu} d x^{\nu} \equiv l^{2}\left(d \sigma^{2}+d \Omega_{3}^{2}\right)
$$

where $d \Omega_{3}^{2}$ corresponds to the metric of 3 dimensional unit sphere, we now include the quantum correction as in (36):

$$
\begin{aligned}
W= & b \int d^{4} x \sqrt{\widetilde{g}} \widetilde{F} A \\
& +b^{\prime} \int d^{4} x\left\{A\left[2 \square^{2}+\widetilde{R}_{\mu \nu} \widetilde{\nabla}_{\mu} \widetilde{\nabla}_{\nu}-\frac{4}{3} \widetilde{R}^{2}+\frac{2}{3}(\widetilde{\nabla} \mu \widetilde{R}) \widetilde{\nabla}_{\mu}\right] A\right. \\
& \left.+\left(\widetilde{G}-\frac{2}{3} \square \widetilde{R}\right) A\right\} \\
& -\frac{1}{12}\left\{b^{\prime \prime}+\frac{2}{3}\left(b+b^{\prime}\right)\right\} \int d^{4} x\left[\widetilde{R}-6 \square A-6\left(\widetilde{\nabla}_{\mu} A\right)\left(\widetilde{\nabla}^{\mu} A\right)\right]^{2} .
\end{aligned}
$$

Note that as typically in Jordan frame there is no non-minimal dilaton coupling with matter we took minimal spinors, i.e. $a=0$. Then one obtains the following brane equations (instead of (65) and (66)):

$$
\begin{aligned}
0= & \frac{l^{4} \mathrm{e}^{4 A}}{8 \pi G}\left\{\left(\frac{8 \alpha}{3 \phi_{0}}-\alpha \phi_{0}\right) \partial_{z} \phi+8 \alpha \partial_{z} A\right. \\
& \left.-\frac{4 \alpha}{3}\left(\frac{6}{l}+\frac{l}{4} \Phi(\phi)\right)\left(\alpha \phi_{0}\right)^{\frac{1}{3}}-\frac{l}{4} \Phi^{\prime}(\phi)(\alpha \phi)^{\frac{4}{3}}\right\} \\
& +b^{\prime}\left(4 \partial_{\sigma}^{4} A-16 \partial_{\sigma}^{2} A\right) \\
& -4\left(b+b^{\prime}\right)\left(\partial_{\sigma}^{4} A+2 \partial_{\sigma}^{2} A-6\left(\partial_{\sigma} A\right)^{2} \partial_{\sigma}^{2} A\right), \\
0= & \frac{48 l^{4}}{16 \pi G} \mathrm{e}^{4 A}\left(\alpha \phi_{0} \partial_{z} A+\frac{\alpha}{3} \partial_{z} \phi-\frac{1}{6}\left(\frac{6}{l}+\frac{l}{4} \Phi(\phi)\right)\left(\alpha \phi_{0}\right)^{\frac{4}{3}}\right) \\
& +\frac{4}{3} a b^{\prime}\left(4 \partial_{\sigma}^{4} A-16 \partial_{\sigma}^{2} A\right) .
\end{aligned}
$$

For $\Phi(\phi)=0$ case, substituting the solution in (60), one finds

$$
\begin{aligned}
0= & \frac{1}{\pi G l}\left\{\sqrt{1+\frac{k l^{2}}{3\left(\alpha \phi_{0}\right)^{\frac{2}{3}} R^{2}}+\frac{l^{2} c^{2}}{24\left(\alpha \phi_{0}\right)^{\frac{8}{3}} R^{8}}}-1\right\}\left(\alpha \phi_{0}\right)^{\frac{4}{3}} R^{4} \\
& +8 b^{\prime}, \\
0= & -\frac{c}{8 \pi G} \\
& +\frac{1}{\pi G l \phi_{0}}\left\{\sqrt{1+\frac{k l^{2}}{3\left(\alpha \phi_{0}\right)^{\frac{2}{3}} R^{2}}+\frac{l^{2} c^{2}}{24\left(\alpha \phi_{0}\right)^{\frac{8}{3}} R^{8}}}-1\right\}\left(\alpha \phi_{0}\right)^{\frac{4}{3}} R^{4} .
\end{aligned}
$$

Combining (75) and (76), one gets 


$$
0=-\frac{c}{8 \pi G}-\frac{8 b^{\prime}}{\phi_{0}}
$$

Eq.(77) has non-trivial solution and can be solved with respect to $\phi_{0}$ :

$$
\phi_{0}=-\frac{64 \pi G b^{\prime}}{c}
$$

In the classical case that $b^{\prime}=0$, there is no solution for $(75)$. Let us define a function $F(R, c)$ as

$$
F(R, c) \equiv \frac{1}{\pi G l}\left\{\sqrt{1+\frac{k l^{2}}{3\left(\alpha \phi_{0}\right)^{\frac{2}{3}} R^{2}}+\frac{l^{2} c^{2}}{24\left(\alpha \phi_{0}\right)^{\frac{8}{3}} R^{8}}}-1\right\}\left(\alpha \phi_{0}\right)^{\frac{4}{3}} R^{4}
$$

It appears in the r.h.s. in $(\sqrt{75})$.

For $k>0$ case, $F(R, c)$ has a minimum at $R=R_{0}$, where $R_{0}$ is defined by

$$
0=\frac{8 k l^{2}}{3\left(\alpha \phi_{0}\right)^{\frac{2}{3}} R_{0}^{2}}+\frac{k^{2} l^{4}}{\left(\alpha \phi_{0}\right)^{\frac{4}{3}} R_{0}^{4}}-\frac{2 l^{2} c^{2}}{3\left(\alpha \phi_{0}\right)^{\frac{8}{3}} R_{0}^{8}} .
$$

When $k>0$, there is only one solution for $R_{0}$. Therefore $F(R, c)$ in the case of $k>0$ (sphere case) is a monotonically increasing function of $R$ when $R>R_{0}$ and a decreasing function when $R<R_{0}$. Since $F(R, c)$ is clearly a monotonically increasing function of $c$, we find for $k>0$ and $b^{\prime}<0$ case that $R$ decreases when $c$ increases if $R>R_{0}$, that is, the non-trivial dilaton makes the radius smaller.

Since one finds

$$
F\left(R_{0}, c\right)=\frac{k l\left(\alpha \phi_{0}\right)^{\frac{2}{3}} R_{0}^{2}}{4 \pi G}
$$

using (79) and (80), Eq.(75) has a solution if

$$
\frac{k l\left(\alpha \phi_{0}\right)^{\frac{2}{3}} R_{0}^{2}}{4 \pi G} \leq-8 b^{\prime} .
$$

That puts again some bounds to the dilaton value. When $|c|$ is small, using (80), one obtains

$$
R_{0}^{4} \sim \frac{2 c^{2}\left(\alpha \phi_{0}\right)^{-\frac{4}{3}}}{3 k^{2} l^{2}}, \quad F\left(R_{0}, c\right) \sim \frac{1}{4 \pi G} \frac{|c|}{\sqrt{3}} .
$$

Therefore Eq.(82) is satisfied for small $|c|$. On the other hand, when $c$ is large, we get

$$
R_{0}^{6} \sim \frac{c^{2}\left(\alpha \phi_{0}\right)^{-\frac{6}{3}}}{4 k}, \quad F\left(R_{0}, c\right) \sim \frac{(k|c|)^{\frac{2}{3}}}{4^{\frac{4}{3}} \pi G} .
$$

Therefore Eq.(82) is not always satisfied and we have no solution for $R$ in (43) for very large $|c|$. 
We now consider the $k<0$ case. When $c=0$, there is no solution for $R$ in (75). Let us define another function $G(R, c)$ as follows:

$$
G(R, c) \equiv 1+\frac{l^{2} c^{2}}{24\left(\alpha \phi_{0}\right)^{\frac{8}{3}} R^{8}}+\frac{k l^{2}}{3\left(\alpha \phi_{0}\right)^{\frac{2}{3}} R^{2}} .
$$

Since $G(R, c)$ appears in the root of $F(R, c)$ in $(79), G(R, c)$ must be positive. Then since

$$
\frac{\partial G(R, c)}{\partial R}=-\frac{l^{2} c^{2}}{3\left(\alpha \phi_{0}\right)^{\frac{8}{3}} R^{9}}-\frac{2 k l^{2}}{3\left(\alpha \phi_{0}\right)^{\frac{2}{3}} R^{3}},
$$

$G(R, c)$ has a minimum

$$
1+\frac{k l^{2}}{4}\left(-\frac{2 k}{c^{2}}\right)^{\frac{1}{3}}
$$

when

$$
R^{6}=-\frac{c^{2}\left(\alpha \phi_{0}\right)^{-\frac{6}{3}}}{2 k}
$$

Therefore if

$$
c^{2} \geq \frac{k^{4} l^{6}}{32}
$$

$F(R, c)$ is real for any positive value of $R$. Since

$$
F(0, c)=\frac{|c|}{\pi G \sqrt{24}}
$$

and when $R \rightarrow \infty$

$$
F(R, c) \rightarrow \frac{k l\left(\alpha \phi_{0}\right)^{\frac{2}{3}} R^{2}}{6 \pi G}<0,
$$

there is a solution $R$ in (75) if

$$
\frac{|c|}{\pi G \sqrt{24}}>-8 b^{\prime}
$$

This is the same bound as in Einstein frame (previous section).

Thus we demonstrated the complete equivalency of quantum induced inflationary (hyperbolic) dilatonic brane-worlds in Einstein and string (Jordan) frames.

Note that Eq.(75) is identical with the corresponding equation (43) in the Einstein frame if we regard $\left(\alpha \phi_{0}\right)^{\frac{1}{3}} R$ as the radius $R_{E}$ in the Einstein frame:

$$
R=\left(\alpha \phi_{0}\right)^{-\frac{1}{3}} R_{E} \text {. }
$$

Then the solution has properties similar to those in the Einstein frame. Since $b^{\prime}$ is order $N$ quantity from (38), Eq.(78) and (93) might tell that the radius $R$ in the Jordan frame is much smaller than the radius $R_{E}$ in the Einstein frame if $N$ is large. In case that the brane is sphere, the brane becomes de Sitter space. Since the rate of the expansion is given by $\frac{1}{R}$ in de Sitter space, the rate might become much larger if compare with that in the Einstein frame when $N$ is large. Thus, even having formal equivalency, the physical interpretation of results obtained in Jordan and Einstein frames may be different. 


\section{BRANE-WORLD BLACK HOLES IN STRING AND EINSTEIN FRAMES}

In analogy with Randall-Sundrum model [1], we now consider the following classical action of the gravity coupled with dilaton $\phi$ in the Einstein frame with Lorentzian signature:

$$
\begin{aligned}
S= & \frac{1}{16 \pi G}\left[\int d^{5} x \sqrt{-g_{(5)}}\left(R_{(5)}-\frac{1}{2} \partial_{\mu} \phi \partial^{\mu} \phi-V(\phi)\right)\right. \\
& \left.-\sum_{i=\text { hid,vis }} \int_{B_{i}} d^{4} x \sqrt{-g_{(4)}} U_{i}(\phi)\right] .
\end{aligned}
$$

Here $B_{\text {hid }}$ and $B_{\text {vis }}$ are branes corresponding to hidden and visible sectors respectively and $U_{i}(\phi)$ corresponds to the vacuum energies on the branes in [1]. One assumes $U(\phi)$ is dilaton dependent and its form is explicitly given later on from the consistency of the equations of motion. The dilaton potential $V(\phi)$ is often given in terms of the superpotential $W(\phi)$ :

$$
V=\left(\frac{\partial W}{\partial \phi}\right)^{2}-\frac{4}{6} W^{2}
$$

We assume again $\phi$ only depends on $z$ and the metric has the following form:

$$
d s^{2}=d z^{2}+\mathrm{e}^{2 A(z)} \tilde{g}_{i j} d x^{i} d x^{j} .
$$

Here $\tilde{g}_{i j}$ is the metric of the Einstein manifold. We also suppose the hidden and visible branes sit on $z=z_{\text {hid }}$ and $z=z_{\text {vis }}$, respectively. Then the equations of motion are given by

$$
\begin{aligned}
& \phi^{\prime \prime}+4 A^{\prime} \phi^{\prime}=\frac{\partial V}{\partial \phi}+\sum_{i=\text { hid,vis }} \frac{\partial U_{i}(\phi)}{\partial \phi} \delta\left(z-z_{i}\right), \\
& 4 A^{\prime \prime}+4\left(A^{\prime}\right)^{2}+\frac{1}{2}\left(\phi^{\prime}\right)^{2} \\
& =-\frac{1}{3} V(\phi)-\frac{2}{3} \sum_{i=\text { hid,vis }} U_{i}(\phi) \delta\left(z-z_{i}\right), \\
& A^{\prime \prime}+4\left(A^{\prime}\right)^{2}=k \mathrm{e}^{-2 A}-\frac{1}{3} V(\phi)-\frac{1}{6} \sum_{i=\text { hid, vis }} U_{i}(\phi) \delta\left(z-z_{i}\right) .
\end{aligned}
$$

Here $^{\prime} \equiv \frac{d}{d z}$. Especially when $k=0$, Eqs. (97-99) have the following first integrals in the bulk:

$$
\phi^{\prime}=\sqrt{2} \frac{\partial W}{\partial \phi}, \quad A^{\prime}=-\frac{1}{3 \sqrt{2}} W .
$$

Near the branes, Eqs. (9799) have the following form :

$$
\phi^{\prime \prime} \sim \frac{\partial U_{i}(\phi)}{\partial \phi} \delta\left(z-z_{i}\right), \quad A^{\prime \prime} \sim-\frac{U_{i}(\phi)}{6} \delta\left(z-z_{i}\right),
$$

or 


$$
2 \phi^{\prime} \sim \frac{\partial U_{i}(\phi)}{\partial \phi}, \quad 2 A^{\prime} \sim-\frac{U_{i}(\phi)}{6}
$$

at $z=z_{i}$. Comparing (102) with $(100)$, we find

$$
U_{\text {hid }}(\phi)=2 \sqrt{2} W(\phi), \quad U_{\text {vis }}(\phi)=-2 \sqrt{2} W(\phi) .
$$

We should note that $k=0$ does not always mean the brane is flat. As well-known, the Einstein equations are given by,

$$
R_{\mu \nu}-\frac{1}{2} g_{\mu \nu} R+\frac{1}{2} \Lambda g_{\mu \nu}=T_{\mu \nu}^{\text {matter }} .
$$

Here $T_{\mu \nu}^{\text {matter }}$ is the energy-momentum tensor of the matter fields. If we consider the vacuum solution where $T_{\mu \nu}^{\text {matter }}=0$, Eq.([104) can be rewritten as

$$
R_{\mu \nu}=\frac{\Lambda}{2} g_{\mu \nu} .
$$

If we put $\Lambda=2 k$, Eq.(105) is nothing but the equation for the Einstein manifold. The Einstein manifolds are not always homogeneous manifolds like flat Minkowski, (anti-)de Sitter space

$$
d s_{4}^{2}=-V(r) d t^{2}+V^{-1}(r) d r^{2}+r^{2} d \Omega^{2}, \quad V(r)=1-\frac{\Lambda}{6} r^{2},
$$

or Nariai space

$$
d s_{4}^{2}=\frac{1}{\Lambda}\left(\sin ^{2} \chi d \psi^{2}-d \chi^{2}-d \Omega^{2}\right) .
$$

but they can be some black hole solutions like Schwarzschild-(anti-)de Sitter black hole

$$
d s_{4}^{2}=-V(r) d t^{2}+V^{-1}(r) d r^{2}+r^{2} d \Omega^{2}, \quad V(r)=1-\frac{\tilde{G}_{4} M}{r}-\frac{\Lambda}{6} r^{2} .
$$

As a special case, one can also consider $k=0$ solution like Schwarzschild black hole,

$$
d s_{4}^{2} \equiv \tilde{g}_{i j} d x^{i} d x^{j}=-\left(1-\frac{\tilde{G}_{4} M}{r}\right) d t^{2}+\frac{d r^{2}}{\left(1-\frac{\tilde{G}_{4} M}{r}\right)}+r^{2} d \Omega^{2} .
$$

In (108) and (109), $M$ is the mass of the black hole on the brane and the effective gravitational constant $G_{4}$ on the 3 -brane (here $d=4$ ) is given by

$$
\frac{1}{G_{4}}=\frac{1}{G} \int_{z_{\text {hid }}}^{z_{\mathrm{vis}}} d z \mathrm{e}^{(d-2) A}
$$

In these solutions, the curvature singularity at $r=0$ has a form of line penetrating the bulk $5 \mathrm{~d}$ universe and the horizon makes a tube surrounding the singularity. The singularity and the horizon connect the hidden and visible branes. These black holes have been discussed in ref. [15]. 
We now consider the Jordan frame, in order to see if singularity supports (or breaks) the equivalency on classical level. Using scale transformation given by (50) and (51) with $D=5$, the action (94) is rewritten as

$$
\begin{aligned}
S= & \frac{1}{16 \pi G} \int d^{5} x \sqrt{g_{(5)}}\left(\alpha \phi R_{(5)}+\frac{4 \alpha}{3 \phi} \nabla_{\mu} \phi \nabla^{\mu} \phi-\frac{\alpha}{2} \phi \nabla_{\mu} \phi \nabla^{\mu} \phi\right. \\
& \left.-V(\phi)(\alpha \phi)^{\frac{5}{3}}\right) \\
& \left.-\sum_{i=\text { hid,vis }} \int_{B_{i}} d^{4} x \sqrt{-g_{(4)}}(\alpha \phi)^{\frac{4}{3}} U_{i}(\phi)\right] .
\end{aligned}
$$

Then if we choose the metric as in (96) in the Jordan frame and $\phi$ only depends on $z$ again, we obtain the following equations instead of (97), (98) and (99),

$$
\begin{aligned}
& \alpha\left(\phi \phi^{\prime \prime}+4 A^{\prime} \phi \phi^{\prime}+\left(\phi^{\prime}\right)^{2}\right) \\
& =\frac{\partial V}{\partial \phi}(\alpha \phi)^{\frac{5}{3}}+\sum_{i=\text { hid,vis }} \frac{\partial U_{i}(\phi)}{\partial \phi}(\alpha \phi)^{\frac{4}{3}} \delta\left(z-z_{i}\right), \\
& \alpha \phi\left(4 A^{\prime \prime}+4\left(A^{\prime}\right)^{2}\right)+\frac{\alpha}{2} \phi\left(\phi^{\prime}\right)^{2}-\frac{4 \alpha}{3 \phi}\left(\phi^{\prime}\right)^{2}+\frac{4 \alpha}{3}\left(\phi^{\prime \prime}+A^{\prime} \phi^{\prime}\right) \\
& \quad=-\frac{1}{3} V(\phi)(\alpha \phi)^{\frac{5}{3}}-\frac{2}{3} \sum_{i=\text { hid,vis }} U_{i}(\phi)(\alpha \phi)^{\frac{4}{3}} \delta\left(z-z_{i}\right), \\
& \alpha \phi\left(A^{\prime \prime}+4\left(A^{\prime}\right)^{2}\right)+\frac{\alpha}{3}\left(\phi^{\prime \prime}+7 A^{\prime} \phi^{\prime}\right) \\
& =k \alpha \phi \mathrm{e}^{-2 A}-\frac{1}{3} V(\phi)(\alpha \phi)^{\frac{5}{3}}-\frac{1}{6} \sum_{i=\text { hid,vis }} U_{i}(\phi)(\alpha \phi)^{\frac{4}{3}} \delta\left(z-z_{i}\right) .
\end{aligned}
$$

If one transforms the above equations to those in the Einstein frame by changing

$$
\begin{aligned}
& A \rightarrow A-\frac{1}{3} \ln (\alpha \phi) \\
& d z \rightarrow(\alpha \phi)^{-\frac{1}{3}} d z \\
& \qquad \begin{array}{c}
\prime \equiv \partial_{z} \rightarrow(\alpha \phi)^{\frac{1}{3}} \partial_{z} \\
\left.\prime \prime=\partial_{z}^{2} \rightarrow(\alpha \phi)^{\frac{2}{3}}\left(\partial_{z}^{2}+\frac{\partial_{z} \phi}{3 \phi} \partial_{z}\right)\right),
\end{array}
\end{aligned}
$$

then Eqs.(97), (98) and (99), which are the corresponding equations in the Einstein frame, are reproduced. Thus we can confirm the equivalence between the Jordan frame and the Einstein frame description of dilatonic brane-world black holes on the classical level. Their physical interpretation may be again different.

\section{DISCUSSION}

In summary, we discussed AdS/CFT induced quantum dilatonic brane-worlds where branes may be flat, de Sitter (inflationary) or Anti-de Sitter Universe. Actually, such objects 
appear in frames of AdS/CFT correspondence [4] as warped compactification of relevant holographic RG flow [5,6]. The role of free parameter (brane tension) is played by effective brane tension produced by conformal anomaly of QFT sitting on the brane. Hence, only brane quantum effects are considered. We compared the construction of such quantum dilatonic brane-worlds in two frames: string and Einstein one. The very nice feature of brane-worlds is discovered: in all examples under consideration the string and Einstein frames are eqiuvalent! This holds to be true also for the number of classical dilatonic brane-world black holes. This is completely different from the case of quantum corrected $4 \mathrm{~d}$ dilatonic gravity (section 2) where de Sitter Universe with decaying dilaton exists in Einstein frame but does not exist in Jordan frame.

Quantum effects may be useful in other aspects of brane-worlds. In particulary, for flat branes the bulk quantum effects (Casimir force) may be estimated 18 20 and used for radion stabilization. Unfortunately, in usual Randall-Sundrum Universe such quantum effects are actually supporting the radion destabilization. Nevertheless, in the case of thermal RandallSundrum scenario 21] such quantum effects may not only stabilize the radion but also may provide the necessary mass hierarchy [21] (at least, for some temperatures). It would be extremely interesting to estimate the bulk quantum effects for dilatonic backgrounds and to understand their role (as well as frame dependence of such Casimir effect) in the creation of dilatonic brane-worlds.

Another interesting line of research is related with account of quantum effects on graviton perturbations around the brane. As is demonstrated in previous section, they may modify the massive graviton modes around hyperbolic brane. Clearly, in other regimes for quantum induced dilatonic (asymptotically) AdS brane more complicated dynamics may be expected.

\section{ACKNOWLEDGEMENTS}

We thank J. Socorro for participation at the early stage of this work. SDO is grateful to L. Randall for useful discussion. The work by O.O., S.D.O. and V.I.T. has been supported in part by CONACyT grant 28454E and that by S.D.O. in part by CONACyT(CP, ref.990356).

\section{APPENDIX A: REMARKS ON GRAVITATIONAL PERTURBATIONS AROUND HYPERBOLIC BRANE}

In [16, [17], the $\mathrm{AdS}_{4}$ branes in $\mathrm{AdS}_{5}$ were discussed and the existence of the massive normalizable mode of graviton was found. In these papers, the tensions of the branes are free parameters but in the case treated in the present paper, the tension is dynamically determined.

Let us study the role of dynamically generated tension in getting of massive graviton modes. Moreover, we consider dilatonic brane-world. We now regard the brane as an object with a tension $U(\phi)$ and assume the brane can be effectively described by the folowing action:

$$
S_{\text {brane }}=-\frac{1}{16 \pi G} \int d^{4} x \sqrt{-g_{(4)}} U(\phi)
$$


If one assumes the metric in the form of (19), then using the Einstein equation, we find

$$
\partial_{z}^{2} A+4\left(\partial_{z} A\right)^{2}=k \mathrm{e}^{-2 A}+\frac{4}{l^{2}}+\frac{\Phi(\phi)}{3}-\frac{U(\phi)}{6} \delta\left(z-z_{0}\right) .
$$

Then at $z=z_{0}$,

$$
\left.\partial_{z} A\right|_{z=z_{0}}=-\frac{U(\phi)}{12} .
$$

For simplicity, we consider the case of the constant dilaton potential $\Phi(\phi)=0$. Comparing (A3) with (41) and (43), one gets

$$
U(\phi)=-\frac{12}{l}+\frac{96 \pi G b^{\prime}}{R^{4}} .
$$

We should note that the tension becomes $R$ dependent due to the quantum correction. In case of AdS brane $k<0$, if no dilaton is included, the boundary equation (43) does not have any solution for $R$. When there is non-trivial dilaton and the parameter $c$ is large enough, Eq.(43) has a solution. If $c$ is very large

$$
R^{4} \sim \frac{c}{\pi G}+8 b^{\prime}
$$

We now consider the perturbation by assuming the metric in the following form:

$$
d s^{2}=\mathrm{e}^{2 \hat{A}(\zeta}\left(d \zeta^{2}+\left(\hat{g}_{\mu \nu}+\mathrm{e}^{-\frac{3}{2} \hat{A}(\zeta)} h_{\mu \nu}\right) d x^{\mu} d x^{\nu}\right) .
$$

By choosing the gauge conditions $h^{\mu}{ }_{\mu}=0$ and $\nabla^{\mu} h_{\mu \nu}=0$, one obtains the following equation

$$
\left(-\partial_{\zeta}^{2}+\frac{9}{4}\left(\partial_{\zeta} \hat{A}\right)^{2}+\frac{3}{2} \partial_{\zeta}^{2} \hat{A}\right) h_{\mu \nu}=m^{2} h_{\mu \nu}
$$

Here $m^{2}$ corresponds to the mass of the graviton on the brane

$$
\left(\square \pm \frac{1}{R^{2}}\right) h_{\mu \nu}=m^{2} h_{\mu \nu}
$$

Here $\square$ is 4- dimensional d'Alembertian constructed on $\hat{g}_{\mu \nu}$ and the $+(-)$ sign corresponds to (anti-)de Sitter brane. Since $-\mathrm{e}^{A} d \zeta=d z=\sqrt{f} d y$ and $\mathrm{e}^{A}=\frac{\sqrt{y}}{l}$, we find, especially for the case of the constant dilaton potential,

$$
\zeta=-\int d y \sqrt{\frac{f(y)}{y}}=-\frac{l^{2}}{2} \int \frac{d y}{\sqrt{y^{3}\left(1+\frac{c^{2} l^{2}}{24 y^{4}}+\frac{k l^{2}}{3 y}\right)}} .
$$

We now consider the case that $c$ is very large, then

$$
f(y) \sim \frac{6 y^{2}}{c^{2}} .
$$


Since $y_{0}=R^{2}$ if there is a brane at $y=y_{0}$, Eq. (A5) can be rewritten as

$$
y_{0}^{2} \sim \frac{c}{\pi G}+8 b^{\prime} .
$$

If we choose $\zeta=0$ when $y=y_{0}$, Eqs.(A9) and (A10) give

$$
|\zeta|=-\frac{1}{|c|} \sqrt{\frac{8}{3}} y^{\frac{3}{2}}+\zeta_{0}, \quad \zeta_{0} \equiv \frac{1}{|c|} \sqrt{\frac{8}{3}} y_{0}^{\frac{3}{2}}>0 .
$$

Note that the brane separates two bulk regions corresponding to $\zeta<0$ and $\zeta>0$, respectively. Since $y$ takes the value in $\left[0, y_{0}\right], \zeta$ takes the value in $\left[-\zeta_{0}, \zeta_{0}\right]$. Since $A=\frac{1}{2} \ln y$, from (A7), one gets

$$
\left(-\partial_{\zeta}^{2}-\frac{1}{4\left(|\zeta|-\zeta_{0}\right)^{2}}-\frac{1}{\zeta_{0}} \delta(\zeta)\right) h_{\mu \nu}=m^{2} h_{\mu \nu}
$$

The zero mode solution with $m^{2}$ of (A13) is given by

$$
h_{\mu \nu}=\sqrt{\zeta_{0}-|\zeta|} .
$$

The general solution of (A13 with $m^{2} \neq 0$ is given by the Bessel functions:

$$
h_{\mu \nu}=a J_{0}\left(m\left(\zeta_{0}-|\zeta|\right)\right)+b N_{0}\left(m\left(\zeta_{0}-|\zeta|\right)\right) .
$$

The coefficients $a$ and $b$ are constants of the integration and they are determined to satisfy the boundary condition

$$
\left.\frac{\partial_{\zeta} h_{\mu \nu}}{h_{\mu \nu}}\right|_{\zeta \rightarrow 0+}=-\frac{1}{2 \zeta_{0}} .
$$

Note that zero mode solution (A14) satisfies this boundary condition (A16). If $b \neq 0$, the solution in (A15) diverges at $\zeta= \pm \zeta_{0}$ and would not be normalizable. If $b=0$, the condition (A16) reduces to

$$
J_{1}\left(m \zeta_{0}\right)=0
$$

that is

$$
m \zeta_{0}=0,3.8317 \ldots, 7.0155 \ldots, \cdots
$$

The non-vanishing solutions for $m^{2}$ give the mass of the massive graviton modes. Thus, these results indicate that $4 \mathrm{~d}$ dilatonic gravity on quantum induced hyperbolic brane may be trapped near the brane.

Since $\zeta_{0}$ is given by $y_{0}$ in (A12) and $y_{0}$ is expressed by (A11), with the help of $b^{\prime}$, which comes from the quantum correction and is negative, the quantum correction makes $\zeta_{0}$ smaller and increases the massive graviton mode mass $m$. It would be of interest to discuss graviton/dilaton perturbations around asymptotically hyperbolic brane in other regimes and to compare the corresponding predictions in different frames. 


\section{REFERENCES}

[1] L. Randall and R. Sundrum, Phys.Rev.Lett. 83 (1999) 3370, hep-th/9905221; Phys.Rev.Lett. 83 (1999) 4690, hep-th/9906064.

[2] A. Chamblin and H.S. Reall, Nucl.Phys. B562 (1999) 133, hep-th/9903225; N. Kaloper, Phys.Rev. D60 (1999) 123506, hep-th/9905210; A. Lukas, B. Ovrut and D. Waldram, Phys.Rev. D61 (2000) 064003, hep-th/9902071; T. Nihei, Phys.Lett. B465 (1999) 81, hep-th/9905487; H. Kim and H. Kim, Phys.Rev. D61 (2000) 064003, hepth/9909053; D. Chung and K. Freese, Phys.Rev. D61 (2000) 023511; J. Garriga and M. Sasaki, Phys.Rev. D62 (2000) 043523, hep-th/9912118; J. Kim and B. Kyae, Phys.Lett. B486 (2000) 165, hep-th/0005139; R. Maartens, D. Wands, B. Bassett and T. Heard, Phys.Rev. D62 (2000) 041301, hep-ph/9912464; S. Kobayashi, K. Koyama and J. Soda, hep-th/0009160; B. McInnes, hep-th/0009087; S. Rama, hep-th/0010121; N. Deger and A. Kaya, hep-th/0010141; L. Mendes and A. Mazumdar, gr-qc/0009017; K. Kashima, hep-th/0010286; H.D. Kim, hep-th/0012091.

[3] P. Binetruy, C. Deffayet and D. Langlois, Nucl.Phys. B565 (2000) 269, hep-th/9905012; J. Cline, C. Grojean and G. Servant, Phys.Rev.Lett. 83 (1999) 4245; E. Flanagan, S. Tye and I. Wasserman, Phys.Rev. D62 (2000) 024011, hep-ph/9909373; C. Csaki, M. Graesser, C. Kolda and J. Terning, Phys.Lett. B462 (1999) 34; P. Kanti, I. Kogan, K. Olive and M. Pospelov, Phys.Lett. B468 (1999) 31; S.Mukohyama, T. Shiromizu and K. Maeda, Phys.Rev. D62 (2000) 024028, hep-th/9912287; K. Behrndt and M. Cvetic, Phys.Lett. B475 (2000) 253, hep-th/9909058; J. Chen, M. Luty and E. Ponton, JHEP 0009 (2000) 012, hep-th/0003067; S. de Alwis, A. Flournoy and N. Irges, hep-th/0004125; R. Gregory, V.A. Rubakov and S. Sibiryakov, Phys.Rev.Lett. 84 (2000) 5928; S. Nojiri and S.D. Odintsov, JHEP 0007(2000) 049, hep-th/0006232; H. Davouddiasl, J. Hewett and T. Rizzo, hep-ph/0006041; P. Binetruy, J.M. Cline and C. Crojean, hep-th/0007029; N. Mavromatos and J. Rizos, hep-th/0008074; I. Neupane, hep-th/0008190; L. Anchordoqui and K. Olsen, hep-th/0008102; K. Akama and T. Hattori, hep-th/0008133; C. Barcelo and M. Visser, gr-qc/0008008; M. Giovannini, hep-th/0011153.

[4] J.M. Maldacena, $\quad$ Adv.Theor.Math.Phys. 2 (1998) 231; E. Witten, Adv.Theor.Math.Phys. 2 (1998) 253; S. Gubser, I. Klebanov and A. Polyakov, Phys.Lett. B428 (1998) 105.

[5] S. Nojiri, S.D. Odintsov and S. Zerbini, Phys.Rev. D62 (2000) 064006, hep-th/0001192; S. Nojiri and S.D. Odintsov, Phys.Lett. B484 (2000) 119, hep-th/0004097.

[6] S.W. Hawking, T. Hertog and H.S. Reall, Phys.Rev. D62 (2000) 043501, hepth/0003052.

[7] S. Nojiri, O. Obregon and S.D. Odintsov, Phys.Rev. D62 (2000) 104003, hepth/0005127; S. Nojiri, S.D. Odintsov and K.E. Osetrin, hep-th/0009059.

[8] B. Geyer and P. M. Lavrov, "Covariant quantization of gauge theories", Leipzig University, to appear.

[9] V. Faraoni, E Gunzig and P. Nardone, Fundamentals of Cosmic Physics 20 (1999)121, gr-qc/9811047; V. Faraoni, Phys.Rev. D59 (1999) 084021.

[10] I.L. Buchbinder, S.D. Odintsov and I.L. Shapiro, "Effective Action in Quantum Gravity", IOP Publishing, Bristol and Philadelphia 1992. 
[11] S. Nojiri and S.D. Odintsov, Phys.Rev. D57 (1998) 2363; S. Nojiri and S.D. Odintsov, Phys.Lett. B426 (1998) 29; B444 (1998) 92; S. Ichinose and S.D. Odintsov, Nucl.Phys. B539 (1999) 634; P. van Nieuwenhuizen, S. Nojiri and S.D. Odintsov, Phys.Rev. D60 (1999) 084014, hep-th/9901119; S. Nojiri and S.D. Odintsov, Int.J.Mod.Phys. A, Reviews, to appear, hep-th/0009216.

[12] B. Geyer, S.D. Odintsov and S. Zerbini, Phys.Lett. B460 (1999) 58; B. Geyer and V. Kovalev, hep-ph/0011102.

[13] A. Starobinsky, Phys.Lett. B91 (1980) 99.

[14] S. Nojiri and S.D. Odintsov, Phys.Lett. B449 (1999) 39, hep-th/9812017; Phys.Rev. D61 (2000) 044014, hep-th/9905200;

[15] S. Nojiri, O. Obregon, S.D. Odintsov and S. Ogushi, Phys.Rev. D62 (2000) 064017

[16] A. Karch and L. Randall, hep-th/0011156.

[17] I.I. Kogan, S. Mouslopoulos and A. Papazoglou, hep-th/0011141.

[18] J. Garriga, O. Pujolas and T. Tanaka, hep-th/0004109.

[19] S. Nojiri, S.D. Odintsov and S. Zerbini, Class.Quant.Grav. 17 (2000) 4855, hepth/0006115.

[20] R. Hofmann, P. Kanti and M. Pospelov, hep-ph/0012213.

[21] I. Brevik, K. Milton, S. Nojiri and S.D. Odintsov, hep-th/0010205. 